\title{
Power law correlations for sediment transport in pressure driven channel flows
}

\author{
N. A. Patankar \\ Department of Mechanical Engineering, Northwestern University, Evanston, IL 60208 \\ D. D. Joseph, J. Wang \\ Department of Aerospace Engineering and Mechanics, University of Minnesota, Minneapolis, MN 55455 \\ R. D. Barree \\ Barree \& Associates LLC, Lakewood, CO 80235 \\ M. Conway, M. Asadi \\ STIM-LAB, Duncan, OK 73534
}

\begin{abstract}
Lift forces acting on particles play a central role in many cases, such as sediment transport, proppant transport in fractured reservoirs, removal of drill cuttings in horizontal drill holes and cleaning of particles from surfaces. We study the problem of lift using 2D direct numerical simulations and experimental data. The lift-off of single particles and many particles in horizontal flows follow laws of similarity, power laws, which may be obtained by plotting simulation data on log-log plots. Data from slot experiments for fractured reservoirs is processed (for the first time) on log-log plots. Power laws with a parameter dependent power emerge as in the case of Richardson-Zaki correlations for bed expansion by drag.
\end{abstract}

\section{Introduction}

Transport of particles in a channel by fluids occurs in variety of settings such as sediment transport, proppant transport in oil reservoirs, removal of drill cuttings etc. Our focus is on the problem of sand or proppant transport in hydraulic fracturing applications.

Hydraulic fracturing is a process often used to increase the productivity of a hydrocarbon well. A slurry of sand or proppants in a fluid is pumped into the well to be stimulated, at sufficient pressure to exceed the horizontal stresses in the rock at reservoir depth. This opens a vertical fracture penetrating from the well bore far into the pay zone. When the pumping pressure is removed, the rigid sand particles act to prop the fracture open. Productivity is enhanced because the proppant-filled fracture offers a higher-conductivity path for oil extraction.

A typical vertical fracture or crack may be $3 \mathrm{~m}$ high, $30 \mathrm{~m}$ long and $2 \mathrm{~cm}$ wide. The diameter of a typical sand grain is $2 \mathrm{~mm}$, so that the crack width to proppant diameter ratio is about 10 . The proppant density is about $2.4 \mathrm{~g} / \mathrm{cc}$. 


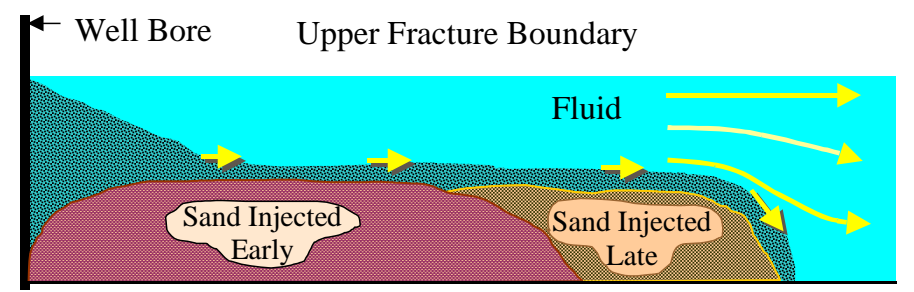

Figure 1. Sand (proppant) transport in a fractured reservoir.

Figure 1 shows the side view of the crack. The fluid-proppant mixture is injected from the well bore. The proppants settle to the bottom as they are dragged forward. A mound of proppant develops and grows until the gap between the top of the crack and the mound reaches an equilibrium value; this value is associated with a critical condition. The velocity in the gap between the mound and the top of the slot increases as the gap size decreases. For velocities below critical the mound gets higher and spreads laterally; for larger velocities proppant is washed out until the new equilibrium height and velocity are established (Kern, Perkins \& Wyant 1959). The accumulation of proppant at the bottom causes good vertical filling to be lost. This reduces well productivity and can also interfere with the fracture growth process by blocking downward extension.

Despite many years of practice and experiments a suitable model for proppant transport or a simulation tool to predict proppant placement is yet to be fully accomplished. We have partnered with STIM-LAB (http://www.stimlab.com), a research laboratory in Duncan, OK, which is supported by a consortium of oil production and oil service companies. STIM-LAB has been collecting data on proppant transport in slots for 15 years.

The physical processes in proppant transport in fractures, described above, are settling and washout. Washout could be by sliding and slipping called the bed-load transport; however, a more efficient transport mechanism is by advection after suspension or fluidization by lift called the suspended-load transport. Lift force plays a central role in the suspension of particles in channel flows. Joseph (2001) proposed that ideas analogous to the Richardson-Zaki (1954) correlation must come into play in problems of slurries, where the particles are fluidized by lift rather than by drag. The problems of fluidization by lift can be decomposed into two separate types of study: (1) single particle studies in which the factors that govern lifting of a heavier- 
than-liquid particle off a wall by a shear flow are identified and (2) many particle studies in which cooperative effects on lift-off are investigated.

Direct numerical simulation (DNS) can be used to extract information implicit in the equations of fluid-particle motion. We have investigated the lift-off of a single particle and many particles in pressure driven flows by 2D DNS (N. A. Patankar, Huang, Ko \& Joseph 2001a, Ko, N. A. Patankar \& Joseph 2001, Joseph, Ocando \& Huang 2001, Choi \& Joseph 2001, N. A. Patankar, Ko, Choi \& Joseph 2001b). We show that the lift-off of single particles and many particles in horizontal flows follow laws of similarity, power laws, which may be obtained by plotting simulation data on log-log plots. Power laws emerge as in the case of Richardson-Zaki correlations for fluidization by drag. Powers laws are expected from the experimental data based on our predictions from 2D numerical simulations. The primary objective of this paper is to process the data from STIM-LAB's experiments in the same way we process data from numerical simulations. The engineering correlations for lift-off can be used to predict proppant placement in the crack.

The fracturing industry makes extensive use of numerical simulation schemes based on models and programmed to run on PC's to guide field operations. These simulations are used to predict how the fracture crack opens and closes and how proppant is transported in the crack. Commercial packages dealing with these problems and propriety packages developed by oil service companies are used extensively. These numerical schemes solve the average equations for the fluid and the proppant phases. The solid and the fluid are considered as inter-penetrating mixtures, which are governed by conservation laws. Interaction between the inter-penetrating phases is modeled. Models for drag and lift forces on the particles must be used for fluidproppant interaction. Models for the drag force on particles in solid-liquid mixtures is a complicated issue and usually rely on the well-known Richardson-Zaki (1954) correlation. Models for lift forces in mixtures are much less well developed than models for drag. Therefore, none of the packages model the all important levitation of proppants by hydrodynamic lift. The power law models we are developing from DNS and experiments may be incorporated in the model-based simulation techniques similar to the model for drag.

The experiments of Segré \& Silberberg (1962) have had a big influence on studies of the fluid mechanics of migration and lift. Eichhorn \& Small (1964) performed experiments to study the lift and drag forces on spheres suspended in a Poiseuille flow. Bagnold (1974) 
experimentally studied the fluid forces on a body in shear flow. Ye \& Roco (1991) experimentally measured the angular velocity of neutrally buoyant particles in a planar Couette flow.

Different analytical expressions for the lift force on a single particle can be found in literature. They are based on perturbing Stokes flow with inertia (e.g. Rubinow \& Keller 1961, Saffman 1965, 1968, Bretherton 1962, Asmolov 1990, McLaughlin 1991, Krishnan \& Leighton 1995 and reference therein) or on perturbing potential flow (e.g. Auton 1987, Drew \& Passman 1999) with a little vorticity. In particular Schonberg \& Hinch (1989), Hogg (1994) and Asmolov (1999) analytically studied the inertial migration of spherical particles in Poiseuille flows. The effect of curvature of the unperturbed velocity profile was found to be important. The domain of parameters for which these analytic expressions are applicable is rather severely restricted. The perturbation analyses are of considerable value because they are analytic and explicit but they are not directly applicable to engineering problems like proppant transport, removal of drill cuttings, sediment transport or even lift-off of heavy single particles.

Dandy \& Dwyer (1990) and Cherukat, McLaughlin \& Dandy (1999) reported computational studies of the inertial lift on a sphere in linear shear flows. Mei (1992) obtained an expression for the lift force by fitting an equation to Dandy \& Dwyer's (1990) data for high Reynolds numbers and Saffman's expression for low Reynolds numbers. The numerical results of Dandy \& Dwyer (1990) are said to be valid for non-rotating spheres. Hence they cannot be applied, strictly speaking, to the case of freely rotating spheres in shear flows. Kurose \& Komori (1999) performed numerical simulations to determine the drag and lift forces on rotating spheres in an unbounded linear shear flow.

Morris \& Brady (1998) studied the migration of non-neutrally buoyant spheres in pressure driven flows of Newtonian fluids. They performed Stokesian dynamic simulations of a monolayer of spheres. These studies are valid in the creeping flow limit. In applications such as the transport of slurries or proppants, the effect of Reynolds number on the lift force is important.

A detailed investigation of the lift-off of single and many particles in channel flows at finite Reynolds numbers is necessary. In section 3 we will review and discuss these results obtained from our 2D direct numerical simulations. New discussion on the implication of these results as compared to the Richardson-Zaki correlation for drag will be presented. 
Shields (1936) reported one of the earliest works on modeling sediment transport. Vanoni (1975) reviews the problem of sedimentation engineering. Sheild's curve (1936) is used to predict the initial motion of sediment particles. For higher fluid flow rates the particles in the moving bed are suspended leading to transport by advection. The problem of determining the flow condition at which the suspension of the sediment begins has been addressed by Bagnold (1966), van Rijn (1984a,b), Sumer (1986) and Celik \& Rodi (1991), among others. There are reasons to investigate the problem further since these studies often provide inconsistent results in determining the condition of the initiation of suspension.

In section 2 we will discuss fluidization by drag and lift. In section 3 we will discuss the results from DNS. The experimental setup will be described in section 4 . The correlations based on experimental data will be presented in section 5. Conclusions will be presented in section 6 .

\section{Analogy between fluidization by drag and lift}
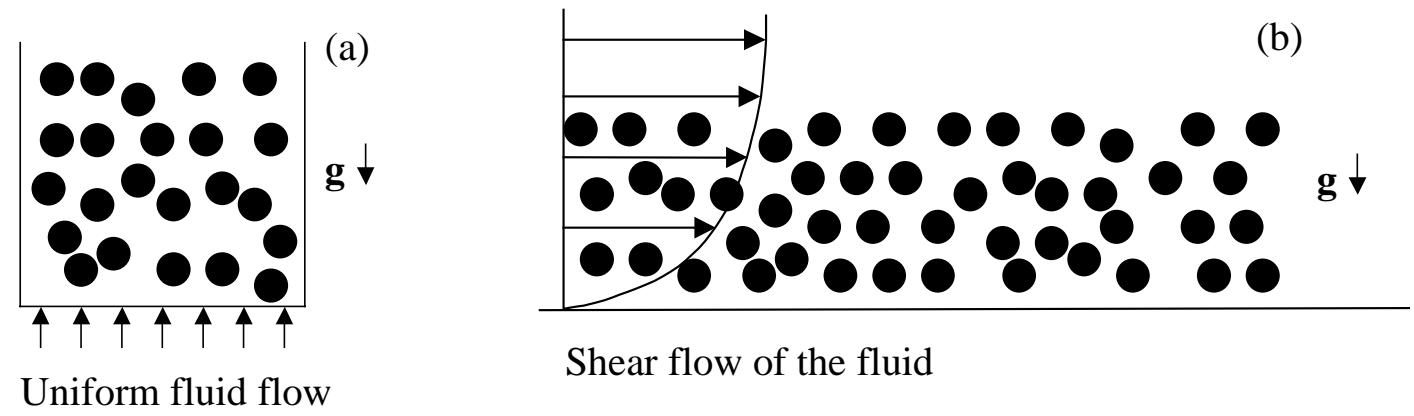

Shear flow of the fluid

Figure 2. (a) Heavy particles fluidized by uniform fluid flow from the bottom of a vertical column. (b) Heavy particles fluidized by lift due to shear flow of the fluid in a horizontal channel. Gravity acts vertically downwards.

Fluidization by drag and shear is depicted in the cartoons in figure 2. In figure 2 a the fluid enters at the bottom of a vertical column at a uniform fluidization velocity. At equilibrium, the drag exerted by the fluid balances the net buoyant weight of the particles. The particle bed acquires a height corresponding to the average particle fraction $\phi$. When the fluidizing velocity is increased the particle bed expands. Richardson \& Zaki (1954) did experiments with different fluids, particles and fluidization velocities. They plotted their data in log-log plots; miraculously this data fell on straight lines whose slope and intercept could be determined. This showed that the variables follow power laws; a theoretical explanation for this outstanding result has not been proposed. After processing the data Richardson \& Zaki (RZ) found that

$$
V_{\phi}=V_{0}[1-\phi]^{n(R)}
$$


where $V_{\phi}$ is the fluidization velocity at the column entrance or the composite velocity. $V_{0}$ is the "blow out" velocity, when $\phi=0$; when $V_{\phi}>V_{0}$ all the particles are blown out of the bed. Clearly $V_{\phi}<V_{0}$ for a fluidized bed. For fluidization columns with large cross section in comparison to the particle size, the RZ exponent $n\left(R_{0}\right)$ depends on the Reynolds number $R_{0}=V_{0} d / \mathrm{v}$ only, where $d$ denotes the particle size e.g. diameter of a spherical particle and $v$ is the kinematic viscosity of the fluid. The power law in the RZ case is an example of what Barenblatt (1996) calls "incomplete self similarity" because the power itself depends on the Reynolds number, a third parameter. Pan, Joseph, Bai, Glowinski \& Sarin (2001) carried out 3D DNS of the fluidization of 1204 spheres and obtained a correlation in agreement with equation 1. The Richardson-Zaki correlation gives different expressions for $n$ for different values of $R_{0}$. In the appendix R. D. Barree presents a way of representing the various expressions for $n$ by a single continuous function.

Equation 1 describes the complicated dynamics of fluidization by drag. The single particle fluidization velocity plays a key role in obtaining the fluidization velocity of concentrated suspensions. An expression for the drag force $F_{d}(1)$ on a single isolated particle in an infinite ambient of the fluid is given by a drag law, e.g.

$$
F_{d}(1)=\left\{\begin{array}{cl}
3 \pi \eta d V_{0}, & \text { laminar } \\
0.055 \pi \rho_{f} d^{2} V_{0}^{2}, & \text { turbulent }
\end{array}\right.
$$

where $\eta$ is the fluid dynamic viscosity, $\rho_{f}$ is the fluid density and spherical particles are considered. In a fluidized bed the total force $F$ acting on a particle is (Foscolo \& Gibilaro 1984, Joseph 1990)

$$
F\left(\varepsilon, R_{0}\right)=F_{d}(\varepsilon)-F_{B}(\varepsilon)
$$

where $\varepsilon$ is the fluid fraction, $F_{d}(\varepsilon)$ is the drag on a single particle in the fluid-particle mixture and $F_{B}(\varepsilon)$ is the effective buoyant weight of a particle in the suspension. We have, $F_{B}(\varepsilon)=V_{p}\left(\rho_{p}\right.$ $\left.\rho_{c}\right) g=\varepsilon V_{p}\left(\rho_{p}-\rho_{f}\right) g=\varepsilon F_{B}(1)$, where $\rho_{p}$ is the particle density, $V_{p}$ is the volume of the particle, $g$ is the gravitational acceleration, $F_{B}(1)$ is the buoyant weight of an isolated particle and $\rho_{c}=\varepsilon \rho_{f}+$ $\phi \rho_{p}$ is the effective or composite density of the fluid-particle mixture. At steady conditions 


$$
\begin{aligned}
& F\left(\varepsilon, R_{0}\right)=0, \\
& \text { i.e. } F_{d}(\varepsilon)=F_{B}(\varepsilon)=\varepsilon F_{B}(1), \\
& \text { i.e. } F_{d}(\varepsilon)=\varepsilon F_{d}(1) .
\end{aligned}
$$

For spherical particles, equations 1, 2 and 4 give

$$
\begin{aligned}
& {\left[\pi d^{3} / 6\right]\left[\rho_{p}-\rho_{f}\right] g=\left\{\begin{array}{cc}
3 \pi \eta d V_{\phi} \varepsilon^{-n\left(R_{0}\right)}, & \text { laminar } \\
0.055 \pi \rho_{f} d^{2} V_{\phi}^{2} \varepsilon^{-2 n\left(R_{0}\right)}, & \text { turbulent }
\end{array}\right.} \\
& \text { or } \\
& R_{G}= \begin{cases}18 \varepsilon^{-n\left(R_{0}\right)} R_{\phi}, & \text { laminar } \\
{\left[\varepsilon^{-n\left(R_{0}\right)} R_{\phi}\right]^{2},} & \text { turbulent }\end{cases}
\end{aligned}
$$

where $R_{G}=\rho_{f}\left[\rho_{p}-\rho_{f}\right] g d^{3} / \eta^{2}$ represents the Reynolds number based on the sedimentation velocity scale $V_{G}=\left[\rho_{p}-\rho_{f}\right] g d^{2} / \eta$ and $R_{\phi}=\rho_{f} V_{\phi} d / \eta$. Equation 5 is another form of the correlation for fluidization by drag and can be written as

$$
R_{G}=a\left(R_{0}\right) R_{\phi}^{p\left(R_{0}\right)} \mathcal{E}^{q\left(R_{0}\right)} .
$$

Figure $2 \mathrm{~b}$ shows the fluidization of particles by shear flow observed in experiments and numerical simulations. At equilibrium the average lift exerted by the fluid should balance the net buoyant weight of the particles. When the applied shear rate is increased the particle bed expands. This is similar to the fluidization by drag where the mechanism for bed expansion is different. Correlations analogous to equation 6 may be expected for fluidization by shear. In that case a Reynolds number based on the applied shear rate should be defined instead of $R_{\phi}$. The prefactor and the exponents may be determined from experimental or numerical data.

In the following sections we will first show that our previous DNS results are in agreement with the above expectation. New discussion based on some of our previous DNS results will be

presented. We will then show that the experimental data obtained from STIM-LAB also give power law correlations.

\section{Direct numerical simulation (DNS) of solid-liquid flows}

We used a numerical method, described in detail by Hu (1996), Hu \& N. A. Patankar (2001) and $\mathrm{Hu}, \mathrm{N}$. A. Patankar \& Zhu (2001), to study the lift-off of a single particle in Newtonian and viscoelastic fluids (N. A. Patankar et. al 2001a, Joseph et. al 2001, Ko et. al 2001). It is an 
Arbitrary-Lagrangian-Eulerian (ALE) numerical method using body-fitted unstructured finite element grids to simulate particulate flows. A closely related numerical method for particulate flows, based on a Chorin (1968) type fractional step scheme, was introduced by Choi (2000). Choi \& Joseph (2001) and N. A. Patankar et. al (2001b) use this scheme to study the fluidization by lift of 300 circular particles in a plane Poiseuille flow by direct numerical simulation.

\subsection{Single particle lift-off}

The principal features of lift-off and levitation to equilibrium in 2D simulations are shown in figure 3. A heavy particle freely translating and rotating in contact with a plane wall in Poiseuille flow is lifted from the wall and suspended in the fluid if the shear Reynolds number $R$ is greater than a critical value. Beyond the critical shear Reynolds number the particle rises from the wall to an equilibrium height at which the buoyant weight $F_{B}$ just balances the hydrodynamic lift $L$ from the fluid. In figure $3, \dot{\gamma}_{w}$ is the shear rate at the wall in the absence of the particle, $U_{p}$ and $\Omega_{p}$ are the translational and angular velocities of the particle, respectively, at steady state and $h_{e}$ is the equilibrium height.

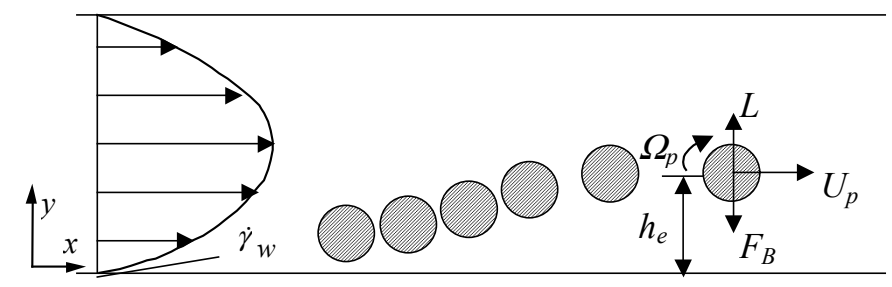

Figure 3. Lift-off and levitation to equilibrium of a single particle.

At steady state the lift force on a freely rotating and translating circular particle in a Poiseuille flow of a Newtonian fluid depends on various parameters,

$$
L=f_{1}\left(\dot{\gamma}_{w}, h_{e}, \rho_{f}, \eta d, H_{1}\right),
$$

where $d$ is the particle diameter and $H_{l}$ is the channel height. At equilibrium $L=\left(\pi d^{2} / 4\right)\left(\rho_{p}\right.$ $\left.\rho_{f}\right) g$. Using this relation and non-dimensionalizing (Buckingham's Pi theorem) we get

$$
R_{G}=f_{2}\left(R, \frac{H_{1}}{d}, \frac{h_{e}}{d}\right)
$$


where the shear Reynolds number $R=\frac{\rho_{f} \dot{\gamma}_{w} d^{2}}{\eta}$ and the gravity Reynolds number or nondimensional lift $R_{G}=\frac{\rho_{f}\left(\rho_{p}-\rho_{f}\right) g d^{3}}{\eta^{2}}$. Freely moving particles in steady flow have zero acceleration. The density ratio $\rho_{p} / \rho_{f}$ vanishes as a parameter when the particle accelerations are zero.

In practical applications the particle acquires some finite separation distance from the wall due to the presence of surface roughness. N. A. Patankar et. al (2001a) and Ko et al. (2001) defined the critical Reynolds number as the minimum shear Reynolds number required to lift a particle to an equilibrium height greater than $0.501 \mathrm{~d}$. They reported that the effect of the channel height on the critical shear Reynolds number for $H_{1} / d>12$ was not significant. At the critical condition, equation 8 implies that $R_{G}$ should be a function of $R$ only. The correlation that N. A. Patankar et al. (2001a) found for the lift-off of a single circular particle in a Newtonian fluid is of the form

$$
R_{G}=a R^{n}, \quad a=2.36, \quad n=1.39 .
$$

This is similar to the form expected from equation 6 and shows that self-similarity lies at the foundation of solid-liquid flows. Similar correlations were found for lift-off in Oldroyd-B fluids by Ko et. al (2001). There the fluid elasticity was an additional parameter.

We found that obtaining a general expression for $R_{G}$ in terms of the $R, h_{e} / d$ and $H_{l} / d$ is not straightforward. This is primarily because of the presence of multiple equilibrium position of a heavy particle at the same Reynolds number, first detected by Choi \& Joseph (2001). It was explained by N. A. Patankar et al. (2001a) to be due to the presence of a turning point bifurcation of the equilibrium solution (figure 4). Turning point bifurcations have also been found in computations of levitation to equilibrium of particles in viscoelastic fluids of Oldroyd-B type (Ko et al. 2001). This implies that the correlation function in equation 8 will not be a single valued monotonous form. We have not observed multiple equilibrium heights of the particle bed in our many particle simulations at the parameters we tested. Hence, many particle correlations for lift in terms of a single particle variable analogous to equation 1 is not preferred. In the next subsection we see that it is more convenient to obtain many particle correlations similar in form to equation 6. 
Multiple stable equilibrium positions are not observed for neutrally buoyant particles. A neutrally buoyant particle has a stable equilibrium position between the channel axis and the wall. This is known since the well-known experiments of Segré \& Silberberg (1962). They found that at low Reynolds number Poiseuille flows, the equilibrium position of a sphere from the pipe axis was found to be 0.6 times the pipe radius.

A general expression for single particle lift force analogous to the drag formula, valid at all Reynolds numbers, has not been obtained yet. Joseph et al. (2001) have suggested that the slip angular velocity discrepancy defined as the difference between the slip angular velocity of a migrating particle and the slip angular velocity at its equilibrium position may be an important variable to model lift. It is positive below the position of equilibrium and negative above it. This discrepancy is the quantity that changes sign above and below the equilibrium position for neutrally buoyant particles, and also above and below the lower equilibrium position for heavy particles. On the other hand the translational slip velocity discrepancy does not change sign. The translational slip velocity is the fluid velocity at the particle center when there is no particle minus the particle velocity. The angular slip velocity is similarly defined.

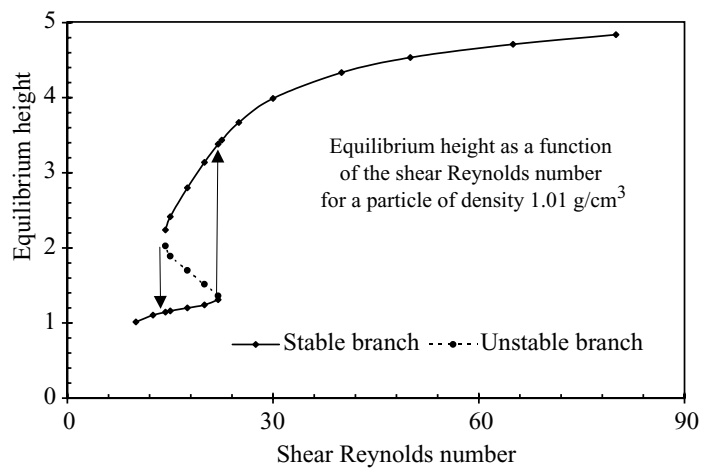

Figure 4. (Patankar, Huang, Ko \& Joseph 2001) Turning point "bifurcations" shown in the equilibrium height (cm) vs. Reynolds number curve. There are two stable branches separated by an unstable branch. The channel height is 12 $\mathrm{cm}$.

\subsection{DNS of levitation to equilibrium of 300 circular particles.}

The transport of a slurry of 300 heavier than liquid particles in a plane pressure driven flow of Newtonian fluids was studied using 2D DNS by Choi \& Joseph (2001) and N. A. Patankar et al. (2001b). Particles are initially placed at the bottom of a periodic channel of height $H_{1}$ in a close packed ordered configuration. The flow is driven by an external pressure gradient. At steady condition, the particle bed reaches a constant height (figure 5). The height of the clear 
fluid region above the particle bed is $H_{2}$ and the average fluid fraction in the particle bed is $\varepsilon$. Similar to equation 8 we expect that

$$
\begin{aligned}
& R_{G}=f_{3}\left(R, \varepsilon, \varepsilon_{\max }, \frac{H_{1}}{d}\right), \\
& \text { where } \\
& \varepsilon=1-\frac{N \pi d^{2}}{4\left[H_{1}-H_{2}\right] l}, \varepsilon_{\text {max }}=1-\frac{N \pi d^{2}}{4 H_{1} l},
\end{aligned}
$$

where $N$ is the number of particles, $l$ is the channel length and $\varepsilon_{\max }$ is the fluid fraction in the particle bed if the particles occupy the entire height of the channel i.e. if $H_{2}=0$.
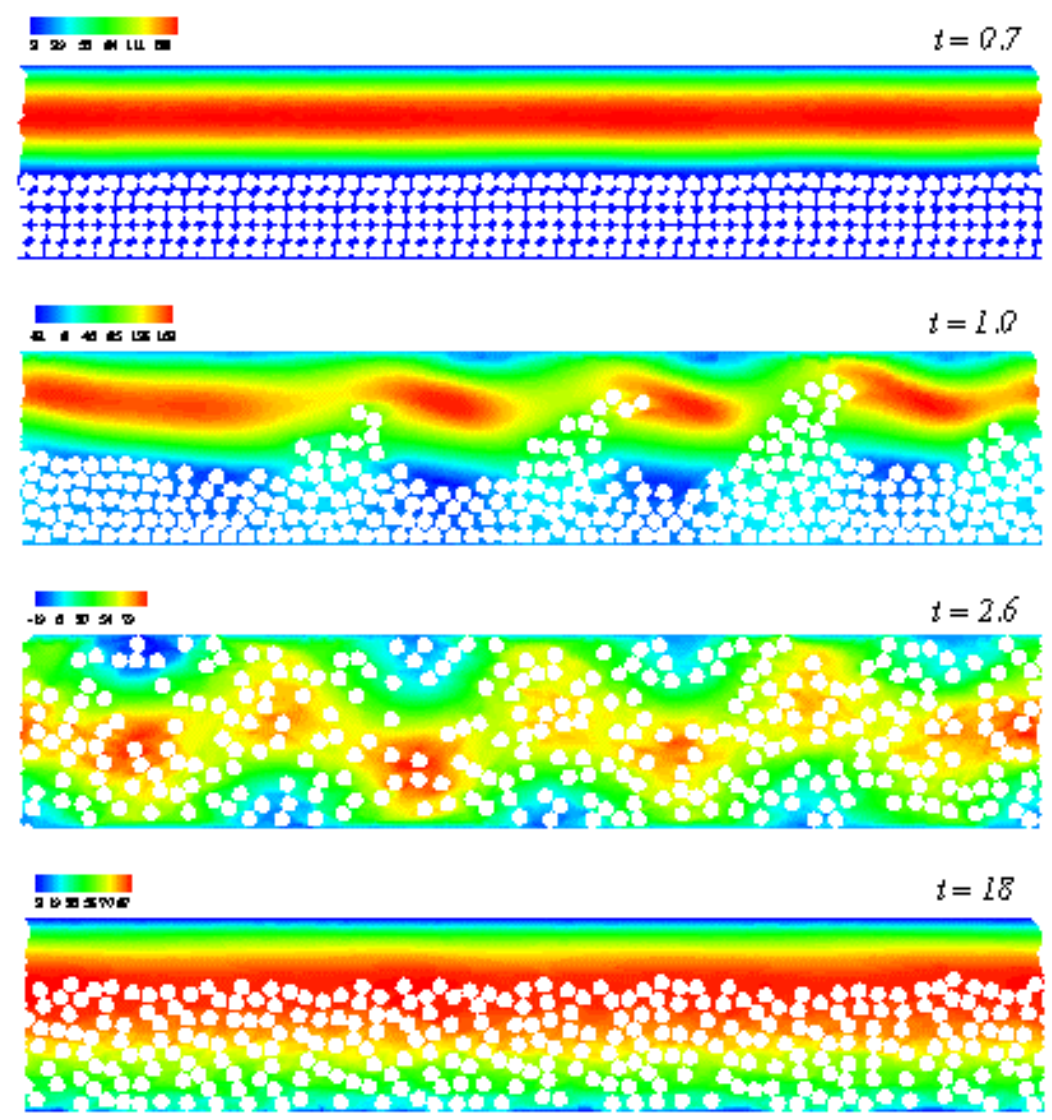

Figure 5. (N. A. Patankar, Ko, Choi \& Joseph 2001b) Lift-off of 300 heavy particles in a plane pressure driven flow of a Newtonian fluid, $\mathrm{Re}=1800$. Contour plot of the horizontal velocity component is shown.

During the simulations $\varepsilon_{\max }$ and $H_{l} / d$ were constant (N. A. Patankar et al. 2001b, Choi \& Joseph 2001). Therefore, $R_{G}$ should be a function of $R$ and $\varepsilon$ only. N. A. Patankar et al. (2001b) obtained the following correlation 


$$
\begin{aligned}
& R_{G}=3.27 \times 10^{-4} \varepsilon^{-9.05} R^{1.249} \\
& \text { or } \\
& R_{G}=3.27 \times 10^{-4}\left[\frac{\left.\varepsilon_{\max }-\frac{H_{2} / H_{1}}{1-H_{2} / H_{1}}\right]^{-9.05} R^{1.249} .}{}\right.
\end{aligned}
$$

The correlation above is of the same form as that expected from equation 6 . This shows that fluidization of slurries by lift also falls into enabling correlations of the RZ type and the above correlation by N. A. Patankar et al. (2001b) could be called a Richardson-Zaki type of correlation for fluidization by lift. Lift results for fluidized slurries are power laws in appropriate dimensionless parameters. These power laws are in the form of engineering type correlations; to use them in applications we need rules for converting two- to three-dimensional results. The goal of our future work is to generate power laws for engineering applications by processing results of simulations in $3 \mathrm{D}$ just as we have done in $2 \mathrm{D}$.

The DNS results are in agreement with the expected power law form in equation 6 from the analogy between fluidization by drag and shear. In the next sections we analyze the experimental data for proppant transport to verify the prediction of power laws from DNS.

\section{Experimental setup}

Kerns, Perkins, and Wyant (1959) reported the earliest experimental investigation of proppant transport in narrow slots. STIM-LAB did more experiments to better understand the processes involved in proppant transport by water and other thin fluids. We have analyzed the data obtained from their experiments. The apparatus used by STIM-LAB was constructed so that the transport of proppant in a horizontally oriented slot could be observed. A schematic of the apparatus is shown in figure 6 . 


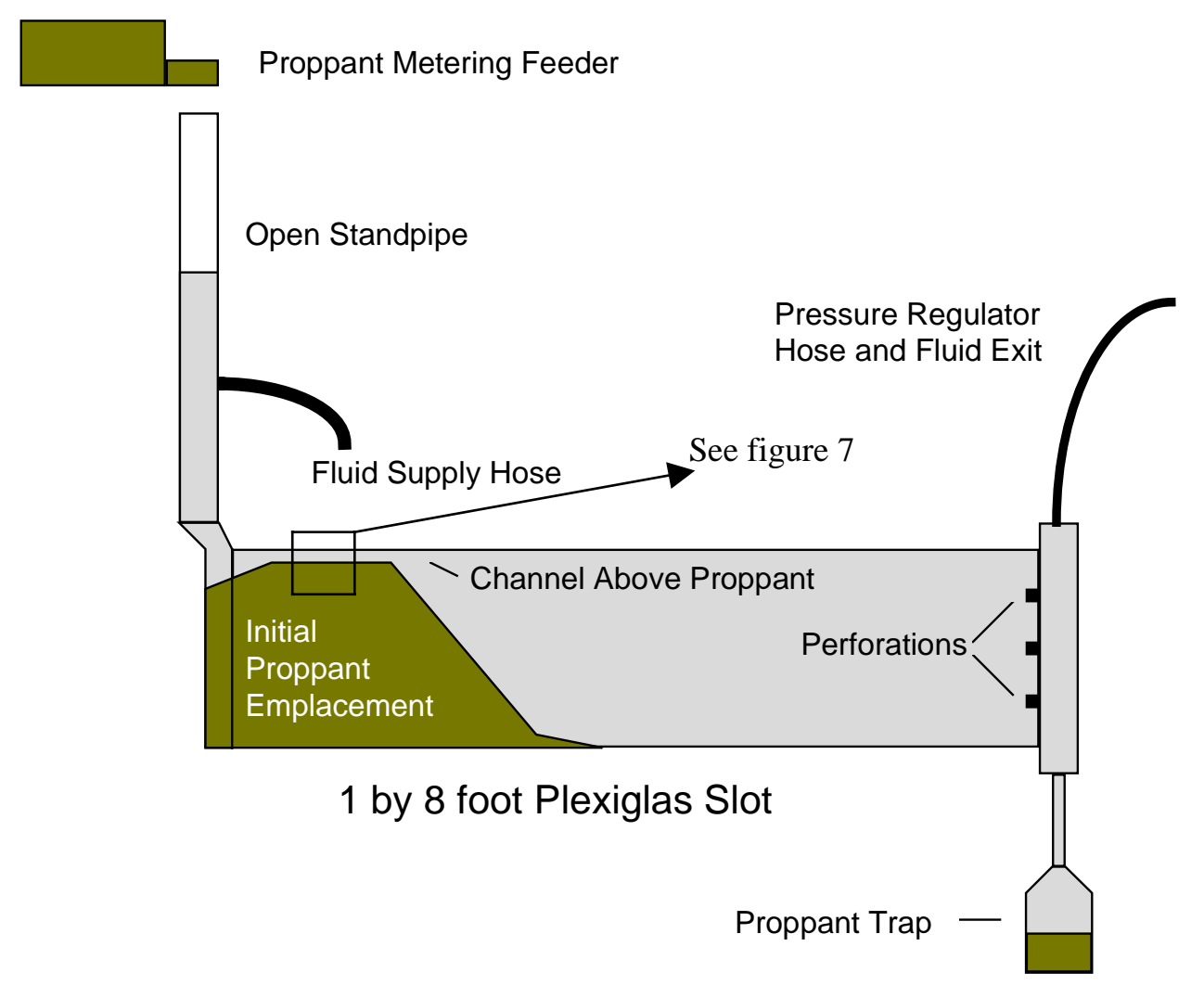

Figure 6. The experimental setup for proppant transport. Proppant and fluid are added at the left where they enter over the full height of the slot. Materials exit at the right through perforations.

Proppant can be added at a constant rate and water flow rate is also constant. Proppant and water enter the $8 \mathrm{~mm}$ wide slot through an open end that is $30.5 \mathrm{~cm}$ tall. The proppant and water then move through the $2.44 \mathrm{~m}$ length of the slot where they exit via three $8 \mathrm{~mm}$ perforations spaced $7.62 \mathrm{~cm}$ apart on the $30.5 \mathrm{~cm}$ tall end of the slot. The proppant and water flow rates were varied, proppants of varying size and density were added and water at different temperatures was used. Observations were recorded and portions of the experiments were video taped.

The evolution of the proppant bed in the experiments is well described in figure 7. The portion shown in figure 7 is marked in figure 6 . In the steady state there is an initial development length (see figure 6) followed by a flat bed region shown in figure 7 and marked in figure 6 . There are three distinct zones in the flat bed region. The bottom part of the bed is immobile; it is a stationary porous medium that supports liquid throughput that might be modeled by Darcy's law. Above the immobile bed is a mobile bed in which particles move by sliding and rolling or advection after suspension or a combination of these modes. Above the mobile bed is the clear fluid zone. At steady state the volumetric fluid flow rate $Q_{f}$ and the volumetric proppant flow rate 
$Q_{p}$ in and out of this region are constant. At steady state, these are equal to the rate at which the fluid and proppant are injected in the slot.

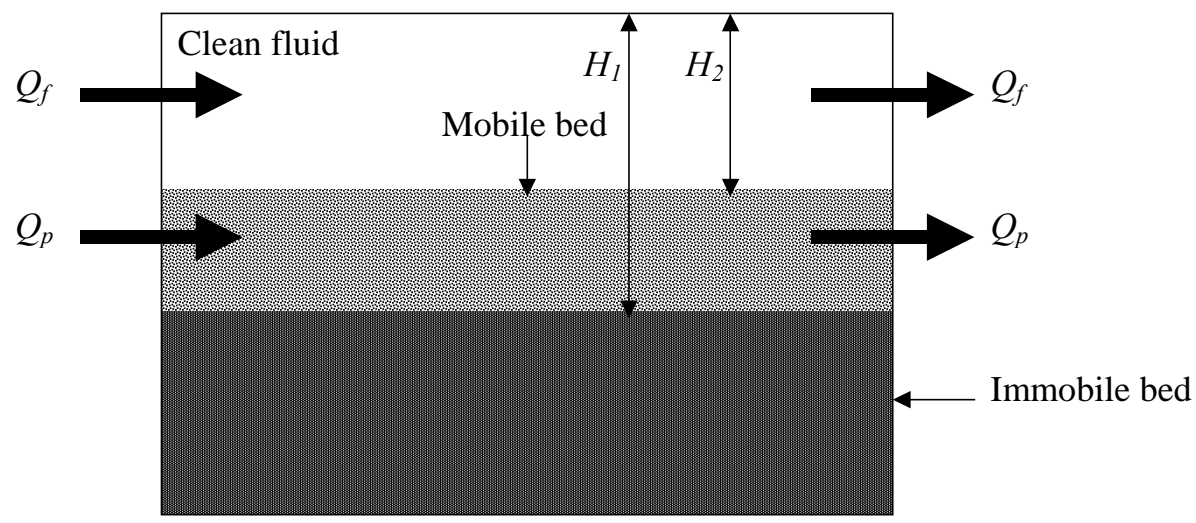

Figure 7. Proppant transport in thin fluids at steady state conditions. In Case 1 only fluid is pumped, $Q_{P}=0, H_{l}=$ $\mathrm{H}_{2}$; the particles are immobile. In Case 2 proppants are also injected, $Q_{P} \neq 0, H_{1} \neq H_{2}$; there is a mobile bed of height $H_{1}-H_{2}$. The channel width $W=8 \mathrm{~mm}$.

STIM-LAB carried out two types of experiments looking at the transport of proppants in thin fluids. In Case 1 only fluid is pumped, $Q_{P}=0, H_{1}=H_{2}$; the particles are immobile. In Case 2 proppants are also injected, $Q_{P} \neq 0, H_{1} \neq H_{2}$; there is a mobile bed of height $H_{1}-H_{2}$. The channel width $W=8 \mathrm{~mm}$. A simplified description of the experiment is that a bed of proppant is eroded by the flow of water. When proppant is not injected as in Case 1, the faster the flow of water the deeper is the channel above the proppants. We are seeking to predict the height above the channel for the given fluid flow rate. In Case 2, we seek to predict both the clear fluid height as well as the mobile bed height as functions of $Q_{f}$ and $Q_{p}$. In the experiments the fluid and the proppant flow rates are controlled and the heights $H_{1}, H_{2}$ are measured.

In the DNS of 300 particles reported by Choi \& Joseph (2001) and N. A. Patankar et al. (2001b) (figure 5), we have a set up similar to that in figure 7 . The value of $H_{1}$ in figure 7 is equivalent to the height of the channel in the simulations. In the simulations, data is obtained for a fixed value of $H_{l} / d$. This is not the case with the experimental data.

\section{Experimental correlations for sediment/proppant transport}

We develop combined correlations for the two cases studied experimentally by STIM-LAB.

5.1 Case 1: $\mathrm{H}_{1}=\mathrm{H}_{2}=\mathrm{H}$ 
This case finds the critical condition of the initial motion of the proppant. Only fluid is injected in the channel. The particle bed is immobile. There is an equilibrium value of $H$ corresponding to a given fluid flow rate. When the fluid flow rate is increased beyond the critical value for a given initial height $H$, the proppants are eroded from the bed and washed out until a new equilibrium height $H$ of the clear fluid region above an immobile bed is achieved for the new flow rate. The data for Case 1 was independently analyzed by D.D. Joseph \& J. Wang and N.A. Patankar. We obtained similar correlations, presented below, from both analyses.

Table 1 gives the data from these experiments. The parameters of this problem are listed below:

Fluid Reynolds number based on channel width $R_{q}=\frac{\rho_{f} \tilde{V} W}{\eta}=\frac{\rho_{f} Q_{f}}{W \eta}$, where $\tilde{V}=\frac{Q_{f}}{W^{2}}$.

Gravity Reynolds number

$$
R_{G}=\frac{\rho_{f}\left[\rho_{p}-\rho_{f}\right] g d^{3}}{\eta^{2}}
$$

Particle diameter/channel width

$d / W$

Height $H /$ channel width

$H / W$.

Figure 8 shows a plot of $R_{q}$ vs. $H / W$ at different values of $R_{G}$.

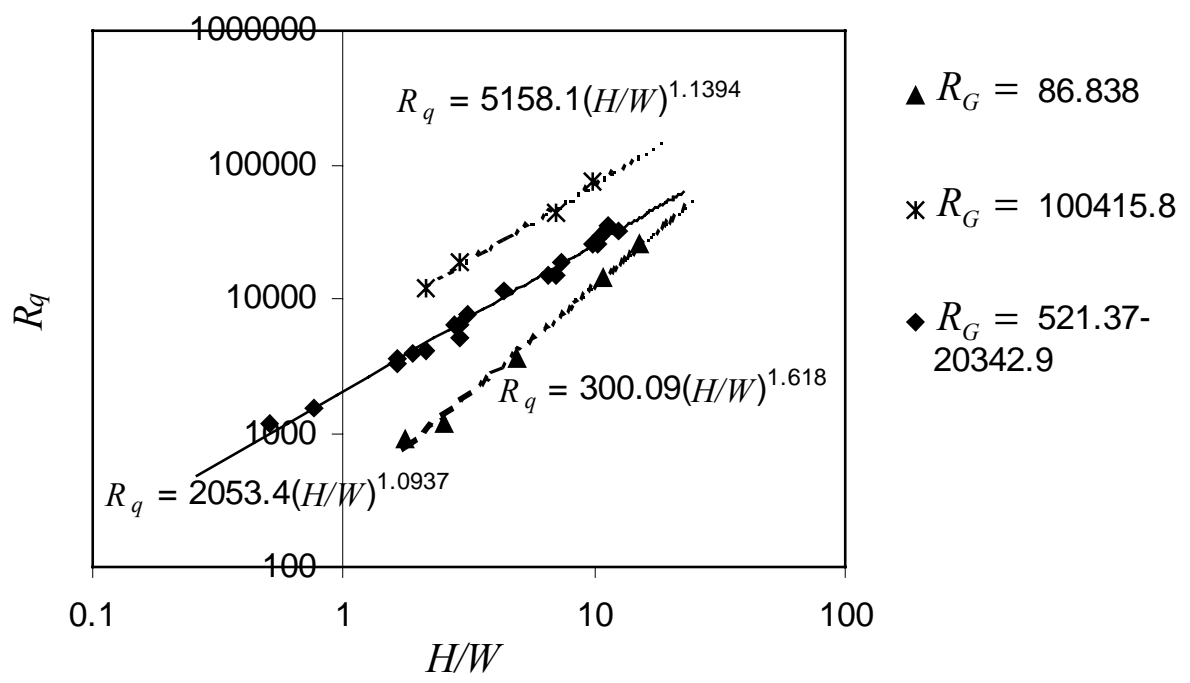

Figure 8. Plot of $R_{q}$ vs. $H / W$ at different values of $R_{G}$ on a logarithmic scale. 


\begin{tabular}{|c|c|c|c|c|c|c|c|c|c|c|}
\hline Proppants & $d(\mathrm{~cm})$ & $\begin{array}{c}H \\
(\mathrm{~cm})\end{array}$ & $\begin{array}{c}\eta \\
(\mathrm{gm} / \mathrm{cm}-\mathrm{s})\end{array}$ & $\begin{array}{c}\rho_{f} \\
(\mathrm{gm} / \mathrm{cc}) \\
\end{array}$ & $\begin{array}{c}Q_{f} \\
\text { (cc/s) }\end{array}$ & $\begin{array}{c}\rho_{p} \\
(\mathrm{gm} / \mathrm{cc})\end{array}$ & $R_{G}$ & $\begin{array}{c}\tilde{V} \\
(\mathrm{~cm} / \mathrm{s})\end{array}$ & $R_{q}$ & $H / W$ \\
\hline & 0.034212 & 1.7 & 0.01115 & 0.999 & 36.778 & 2.65 & 521.1645 & 58.37416 & 4184.12 & 2.141732 \\
\hline $60 / 40$ & 0.034212 & 2.3 & 0.01115 & 0.999 & 58.289 & 2.65 & 521.1645 & 92.51649 & 6631.36 & 2.897638 \\
\hline \multirow[t]{3}{*}{ Brady } & 0.034212 & 5.6 & 0.01115 & 0.999 & 133.295 & 2.65 & 521.1645 & 211.5662 & 15164.55 & 7.055118 \\
\hline & 0.034212 & 7.8 & 0.01115 & 0.999 & 232.588 & 2.65 & 521.1645 & 369.1644 & 26460.80 & 9.826772 \\
\hline & 0.056043 & 2.3 & 0.01115 & 0.999 & 46.556 & 2.65 & 2290.822 & 73.89383 & 5296.48 & 2.897638 \\
\hline $20 / 40$ & 0.056043 & 5.2 & 0.01115 & 0.999 & 133.106 & 2.65 & 2290.822 & 211.2663 & 15142.90 & 6.551181 \\
\hline \multirow[t]{2}{*}{ Ottawa } & 0.056043 & 8.2 & 0.01115 & 0.999 & 227.542 & 2.65 & 2290.822 & 361.1554 & 25886.49 & 10.33071 \\
\hline & 0.06 & 1.4 & 0.01115 & 0.999 & 7.885 & 1.05 & 86.83778 & 12.5151 & 897.05 & 1.76378 \\
\hline $20 / 40$ & 0.06 & 2 & 0.01115 & 0.999 & 10.409 & 1.05 & 86.83778 & 16.5212 & 1184.19 & 2.519685 \\
\hline Light & 0.06 & 3.9 & 0.01115 & 0.999 & 31.92 & 1.05 & 86.83778 & 50.66353 & 3631.42 & 4.913386 \\
\hline \multirow[t]{3}{*}{ Beads } & 0.06 & 8.5 & 0.01115 & 0.999 & 128.438 & 1.05 & 86.83778 & 203.8572 & 14611.89 & 10.70866 \\
\hline & 0.06 & 12 & 0.01115 & 0.999 & 226.217 & 1.05 & 86.83778 & 359.0523 & 25735.84 & 15.11811 \\
\hline & 0.094946 & 1.5 & 0.01 & 0.998 & 31.542 & 2.73 & 14513.72 & 50.06356 & 3997.08 & 1.889764 \\
\hline $16 / 20$ & 0.094946 & 2.2 & 0.01 & 0.998 & 50.467 & 2.73 & 14513.72 & 80.10138 & 6395.30 & 2.771654 \\
\hline \multirow[t]{2}{*}{ Carbolite } & 0.094946 & 9.9 & 0.01 & 0.998 & 258.642 & 2.73 & 14513.72 & 410.5174 & 32775.74 & 12.47244 \\
\hline & 0.094946 & 1.7 & 0.00378 & 0.972 & 36.778 & 2.73 & 100415.8 & 58.37416 & 12008.41 & 2.141732 \\
\hline $16 / 20$ & 0.094946 & 2.3 & 0.00378 & 0.972 & 58.289 & 2.73 & 100415.8 & 92.51649 & 19031.98 & 2.897638 \\
\hline \multirow[t]{3}{*}{ Carbolite } & 0.094946 & 5.6 & 0.00378 & 0.972 & 133.295 & 2.73 & 100415.8 & 211.5662 & 43522.25 & 7.055118 \\
\hline & 0.094946 & 7.8 & 0.00378 & 0.972 & 232.588 & 2.73 & 100415.8 & 369.1644 & 75942.48 & 9.826772 \\
\hline & 0.088437 & 0.4 & 0.01115 & 0.999 & 10.535 & 3.45 & 13363.76 & 16.72119 & 1198.53 & 0.503937 \\
\hline $16 / 30$ & 0.088437 & 0.6 & 0.01115 & 0.999 & 13.878 & 3.45 & 13363.76 & 22.02721 & 1578.85 & 0.755906 \\
\hline \multirow[t]{4}{*}{ Banrite } & 0.088437 & 1.3 & 0.01115 & 0.999 & 29.145 & 3.45 & 13363.76 & 46.25904 & 3315.71 & 1.637795 \\
\hline & 0.088437 & 3.5 & 0.01115 & 0.999 & 100.681 & 3.45 & 13363.76 & 159.8012 & 11454.08 & 4.409449 \\
\hline & 0.088437 & 8.3 & 0.01115 & 0.999 & 261.796 & 3.45 & 13363.76 & 415.5234 & 29783.50 & 10.45669 \\
\hline & 0.109021 & 1.3 & 0.01015 & 0.998 & 28.955 & 2.65 & 20342.9 & 45.95747 & 3615.03 & 1.637795 \\
\hline $12 / 20$ & 0.109021 & 2.5 & 0.01015 & 0.998 & 62.137 & 2.65 & 20342.9 & 98.62404 & 7757.81 & 3.149606 \\
\hline \multirow[t]{2}{*}{ Badger } & 0.109021 & 5.8 & 0.01015 & 0.998 & 155.185 & 2.65 & 20342.9 & 246.3101 & 19374.85 & 7.307087 \\
\hline & 0.109021 & 9 & 0.01015 & 0.998 & 290.814 & 2.65 & 20342.9 & 461.5809 & 36308.13 & 11.33858 \\
\hline
\end{tabular}

Table 1: Data from experiments on the initiation of sediment motion (Case 1).

From figure 8 we get the following form for the correlation of the initiation of sediment motion

$$
R_{q}=a[H / W]^{n},
$$


where $a$ and $n$ are function of $R_{G}$. We see that $a$ and $n$ may be regarded as constants for $521.37 \leq$ $R_{G} \leq$ 20342.9. The values of $a$ and $n$ listed in figure 8 are plotted in figures $9 \mathrm{a}$ and $9 \mathrm{~b}$, respectively.

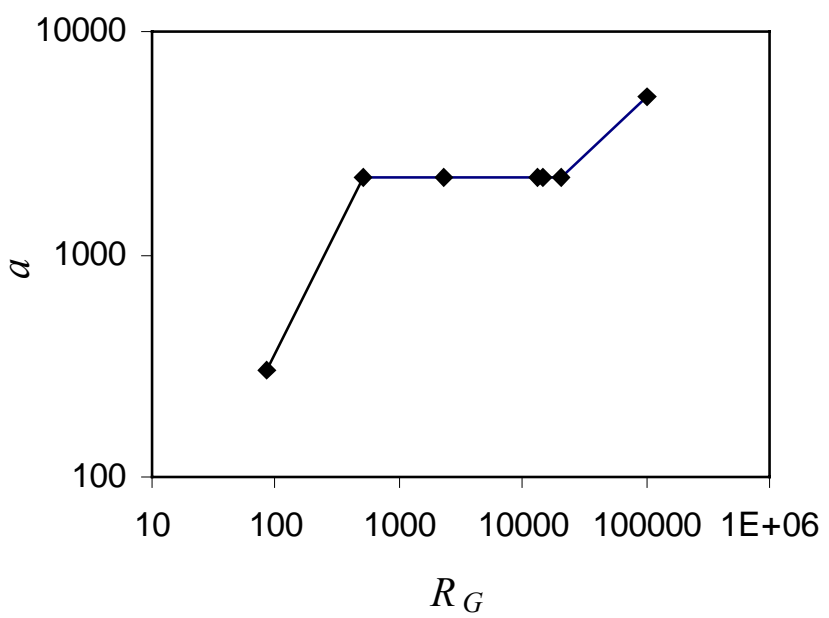

Figure 9(a). Plot of $a$ vs. $R_{G}$ on a logarithmic scale.

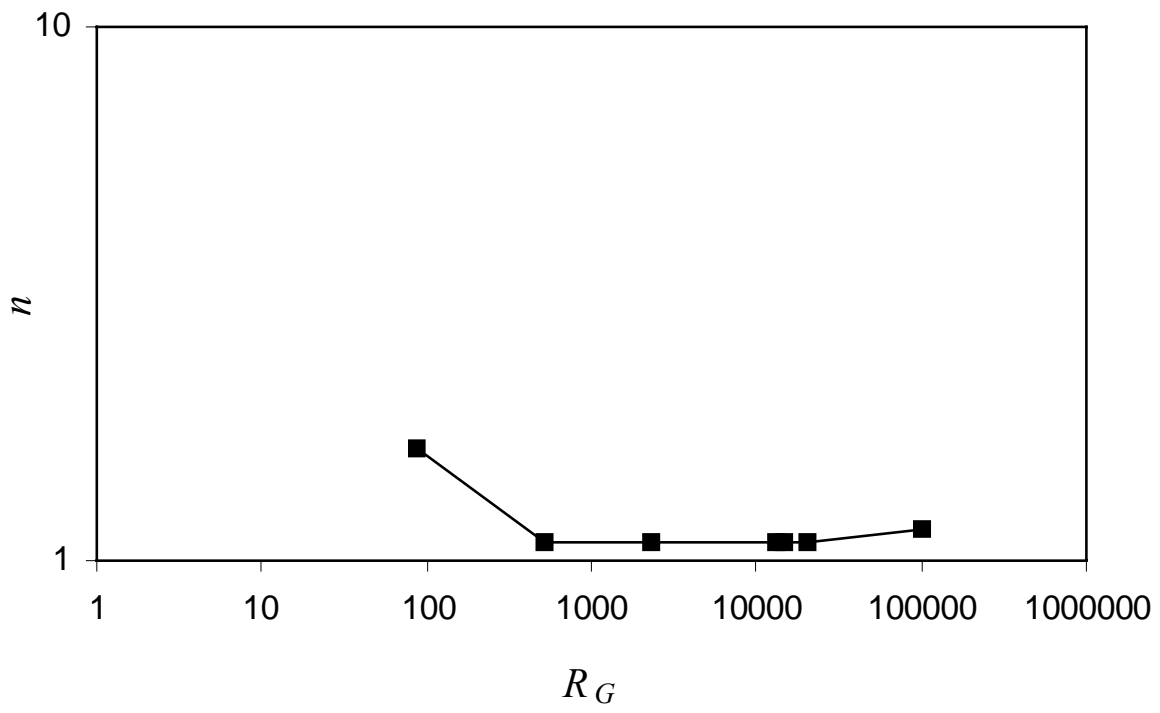

Figure 9(b). Plot of $n$ vs. $R_{G}$ on a logarithmic scale.

We define an effective Reynolds number as 


$$
R_{e f f}=R_{q}[W / H]^{n}
$$

where, the values of $n$ are given in figure 8 and plotted in figure $9 \mathrm{~b}$. This leads to the following expression for the critical effective Reynolds number $R_{\text {eff }}^{c r}$ for the initiation of bed motion

$$
R_{\text {eff }}^{c r}=a\left(R_{G}\right)
$$

The value of $a$ is given in figure 8 and plotted in figure 9 a. $R_{\text {eff }}$ may be regarded as a Reynolds number that accounts for the effect of the side walls of the channel.

Shield's (1936) curve also gives the critical condition for the initiation of sediment motion. The Shields parameter $S$ is defined as: $S=\frac{\tau}{\left[\rho_{p}-\rho_{f}\right] g d}$, where $\tau$ is a measure of the shear stress on the particle bed. If we take $\tau=\eta \tilde{V} / W$, then $S=\frac{\eta \tilde{V}}{\left[\rho_{p}-\rho_{f}\right] g d^{2}}=\frac{R_{q}[d / W]}{R_{G}}$. From the Shield's (1936) curve one obtains (see also, Vanoni 1975) $S=f_{s}\left(\sqrt{R_{q}}[d / W]\right)$. Equation 14, applicable for proppant transport in narrow channels has $W / H$ as another parameter.

\subsection{Case 2: $\mathrm{H}_{1} \neq \mathrm{H}_{2}$}

There are two sets of data for Case 2:

1. 20/40 Ottawa sand: The parameters are given in Table 2 .

\begin{tabular}{|c|c|}
\hline$\rho_{p}$ & $2.65 \mathrm{gm} / \mathrm{cc}$ \\
\hline$d$ & $0.06 \mathrm{~cm}$ \\
\hline$\rho_{f}$ & $1 \mathrm{gm} / \mathrm{cc}$ \\
\hline$\eta$ & $0.01 \mathrm{gm} / \mathrm{cm}-\mathrm{s}$ \\
\hline$W$ & $0.8 \mathrm{~cm}$ \\
\hline$R_{G}$ & 3496 \\
\hline
\end{tabular}

Table 2. Parameters for experiments with 20/40 Ottawa sand in Case 2.

2. 16/30 Carbolite sand: The parameters are given in Table 3 . 


\begin{tabular}{|c|c|}
\hline$\rho_{p}$ & $2.71 \mathrm{gm} / \mathrm{cc}$ \\
\hline$d$ & $0.09 \mathrm{~cm}$ \\
\hline$\rho_{f}$ & $1 \mathrm{gm} / \mathrm{cc}$ \\
\hline$\eta$ & $0.01 \mathrm{gm} / \mathrm{cm}-\mathrm{s}$ \\
\hline$W$ & $0.8 \mathrm{~cm}$ \\
\hline$R_{G}$ & 12229 \\
\hline
\end{tabular}

Table 3. Parameters for experiments with 16/30 Carbolite sand in Case 2.

In this case the fluid and the proppant are both injected at a specified volumetric flow rate. Since the proppants are injected, there is a moving particle bed in the channel. At equilibrium there is a clear fluid region of height $H_{2}$ above a moving bed of height $H_{1}-H_{2}$. The experimental data are given in tables $4 \& 5$.

First we wish to obtain a correlation for the fluid Reynolds number $R_{q}$ in terms of $R_{G}$ and $\varepsilon$. It is more convenient to obtain experimental correlations in terms of $H_{1}$ and $H_{2}$ instead of $\varepsilon$. Power laws in terms of $H_{1} / H_{2}$ were also obtained from numerical simulations (equation 11).

\begin{tabular}{ccccccc}
\hline $\begin{array}{c}H_{I} \\
\mathrm{~cm}\end{array}$ & $\begin{array}{c}H_{2} \\
\mathrm{~cm}\end{array}$ & $\begin{array}{c}Q_{p} \\
\mathrm{cc} / \mathrm{s}\end{array}$ & $\begin{array}{c}Q_{f} \\
\mathrm{cc} / \mathrm{s}\end{array}$ & $\begin{array}{c}Q=Q_{p}+Q_{f} \\
\mathrm{cc} / \mathrm{s}\end{array}$ & $Q_{p} / Q$ & $Q_{f} / Q$ \\
\hline 2.3 & 0.8 & 40 & 244.1 & 284.1 & 0.140795 & 0.859205 \\
2.6 & 0.7 & 45.7 & 242.9 & 288.6 & 0.158351 & 0.841649 \\
2.3 & 1 & 28.6 & 250.4 & 279 & 0.102509 & 0.897491 \\
2.4 & 1.5 & 11.4 & 249.8 & 261.2 & 0.043645 & 0.956355 \\
3 & 2.1 & 11.4 & 313.5 & 324.9 & 0.035088 & 0.964912 \\
2.9 & 1.5 & 34.3 & 304.7 & 339 & 0.10118 & 0.89882 \\
3.1 & 2.3 & 11.4 & 314.8 & 326.2 & 0.034948 & 0.965052 \\
3 & 1.4 & 45.7 & 303.4 & 349.1 & 0.130908 & 0.869092 \\
3 & 1.5 & 40 & 305.3 & 345.3 & 0.115841 & 0.884159 \\
2.9 & 1.6 & 28.6 & 306 & 334.6 & 0.085475 & 0.914525 \\
2.8 & 1.7 & 22.8 & 306 & 328.8 & 0.069343 & 0.930657 \\
3.1 & 2 & 17.1 & 315.4 & 332.5 & 0.051429 & 0.948571 \\
3.5 & 2.9 & 5.7 & 314.2 & 319.9 & 0.017818 & 0.982182 \\
4.1 & 3.6 & 2.9 & 313.5 & 316.4 & 0.009166 & 0.990834 \\
5.1 & 5 & 1.4 & 312.9 & 314.3 & 0.004454 & 0.995546 \\
5.8 & 5.7 & 0.4 & 311.6 & 312 & 0.001282 & 0.998718 \\
\hline
\end{tabular}

Table 4. Experimental data for Case 2 with Ottawa sand. 


\begin{tabular}{ccccccc}
\hline$H_{l}$ & $H_{2}$ & $Q_{p}$ & $Q_{f}$ & $\begin{array}{c}\text { = } Q_{p}+Q_{f} \\
\mathrm{cc} / \mathrm{s}\end{array}$ & $Q_{p} / Q$ & $Q_{f} / Q$ \\
$\mathrm{~cm}$ & $\mathrm{~cm}$ & $\mathrm{cc} / \mathrm{s}$ & $\mathrm{cc} / \mathrm{s}$ & & \\
\hline 2 & 1.2 & 11.2 & 180.4 & 191.6 & 0.058455 & 0.941545 \\
2.1 & 1.2 & 16.8 & 180.4 & 197.2 & 0.085193 & 0.914807 \\
1.7 & 0.5 & 22.3 & 180.4 & 202.7 & 0.110015 & 0.889985 \\
1.9 & 0.6 & 27.9 & 180.4 & 208.3 & 0.133941 & 0.866059 \\
1.9 & 0.4 & 33.5 & 180.4 & 213.9 & 0.156615 & 0.843385 \\
1.7 & 0.2 & 44.7 & 180.4 & 225.1 & 0.198578 & 0.801422 \\
2.4 & 1.5 & 5.6 & 192.4 & 198 & 0.028283 & 0.971717 \\
2.8 & 2.1 & 2.8 & 193.7 & 196.5 & 0.014249 & 0.985751 \\
3 & 2.8 & 1.4 & 193.7 & 195.1 & 0.007176 & 0.992824 \\
3.3 & 2.8 & 0.7 & 195.6 & 196.3 & 0.003566 & 0.996434 \\
4.3 & 3.9 & 0.3 & 195.6 & 195.9 & 0.001531 & 0.998469 \\
2.9 & 1.6 & 22.3 & 306.6 & 328.9 & 0.067802 & 0.932198 \\
2.9 & 2 & 11.2 & 307.2 & 318.4 & 0.035176 & 0.964824 \\
3.3 & 2.6 & 5.6 & 306 & 311.6 & 0.017972 & 0.982028 \\
4 & 3.2 & 2.8 & 309.7 & 312.5 & 0.00896 & 0.99104 \\
4 & 3.2 & 1.4 & 311 & 312.4 & 0.004481 & 0.995519 \\
4.2 & 3.5 & 0.7 & 311 & 311.7 & 0.002246 & 0.997754 \\
5.3 & 5 & 0.3 & 316 & 316.3 & 0.000948 & 0.999052 \\
3.1 & 1 & 44.7 & 315.4 & 360.1 & 0.124132 & 0.875868 \\
3 & 1.2 & 39.1 & 314.2 & 353.3 & 0.110671 & 0.889329 \\
3 & 1.3 & 33.5 & 308.5 & 342 & 0.097953 & 0.902047 \\
3 & 1.3 & 27.9 & 310.5 & 338.4 & 0.082447 & 0.917553 \\
2.9 & 1.6 & 22.3 & 309.1 & 331.4 & 0.06729 & 0.93271 \\
3 & 1.8 & 16.8 & 312.1 & 328.9 & 0.051079 & 0.948921 \\
3.2 & 2.1 & 11.2 & 318.6 & 329.8 & 0.03396 & 0.96604 \\
3.4 & 2.6 & 5.6 & 321.1 & 326.7 & 0.017141 & 0.982859 \\
3.5 & 2.9 & 4.2 & 316 & 320.2 & 0.013117 & 0.986883 \\
\hline & & & & & &
\end{tabular}

Table 5. Experimental data for Case 2 with Carbolite sand.

A correlation obtained for Case 2 should reduce to the correlation for Case 1 when $H_{1}=H_{2}$. From Case 1 we see that when $R_{G}=3496$ (Ottawa) and 12229 (Carbolite) the critical effective Reynolds number $R_{\text {eff }}^{c r}=2053.4$ and $n=1.0937$. We define an effective Reynolds number at the fluid proppant-bed interface as $R_{\text {eff }}=R_{q}\left(W / H_{2}\right)^{1.0937}$ for the Carbolite and Ottawa data. This is consistent with the definition of $R_{\text {eff }}$ in equation 13 since $\mathrm{H}_{2}$ is the height of the clear fluid region. Figure 10 shows a plot of $R_{\text {eff }}$ vs. $\ln \left(H_{1} / H_{2}\right)$ for the combined Carbolite and Ottawa data. 


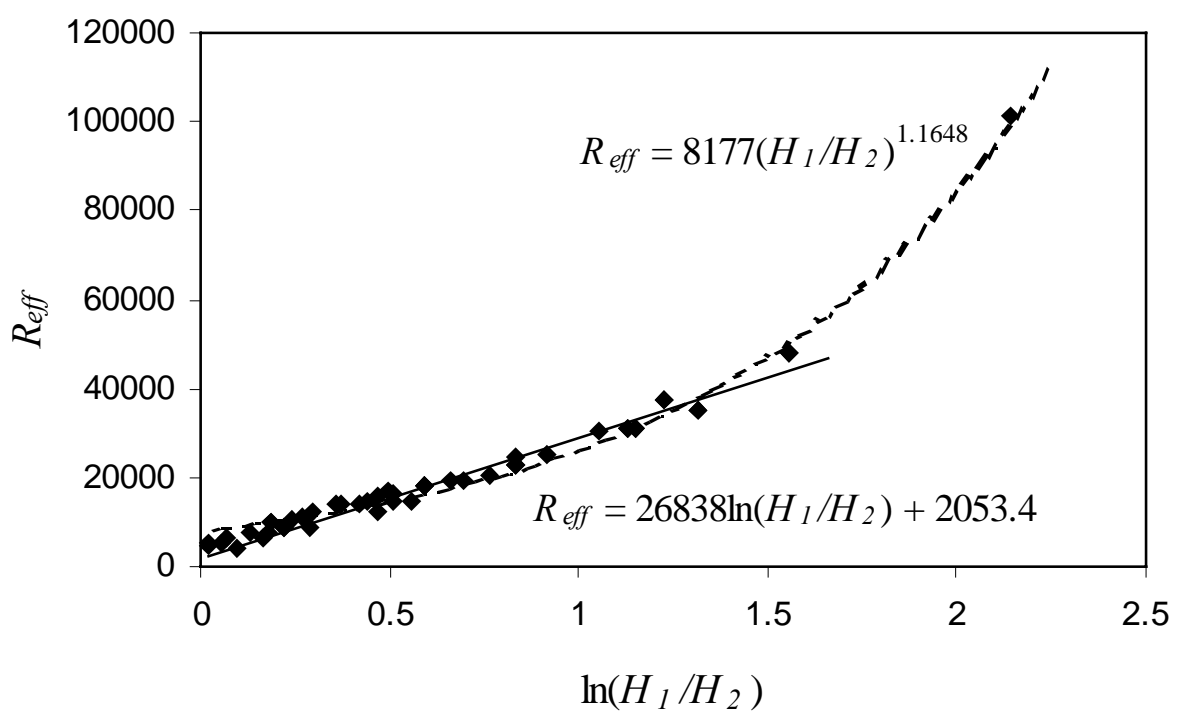

Figure 10. Plot of $R_{\text {eff }}$ vs. $\ln \left(H_{1} / H_{2}\right)$ for the combined Carbolite and Ottawa data.

Two regimes are observed for the given values of $R_{G}$ :

Regime 1: $\quad R_{\text {eff }}-R_{\text {eff }}^{c r}=26838 \ln \left(H_{1} / H_{2}\right)$.

Regime 2:

$$
R_{\text {eff }}=8177\left(H_{1} / H_{2}\right)^{1.1648} .
$$

Note that the y-intercept is taken to be equal to $R_{\text {eff }}^{c r}$. Regime 1 shows logarithmic behavior whereas regime 2 shows power law behavior. When $H_{1} / H_{2}=1$, we recover the correlation in equation 14. Thus equations $15 \& 16$ represent a combined correlation for the data from Cases 1 $\& 2$. Regimes 1 and 2 have an overlap region; we may estimate the transition to begin at $R_{\text {eff }}=$ 20000.

The following general form of the correlation may be proposed:

$$
\left(\frac{\rho_{f} Q_{f}}{H_{2} \eta}\right)\left(W / H_{2}\right)^{n-1}-a\left(R_{G}\right)=26838 \ln \left(H_{1} / H_{2}\right) ; \text { regime } 1,
$$

and

$$
\left(\frac{\rho_{f} Q_{f}}{H_{2} \eta}\right)\left(W / H_{2}\right)^{n-1}=8177\left(H_{1} / H_{2}\right)^{1.1648} ; \text { regime } 2 .
$$

Equations $15 \& 16$ can also be represented by a single equation using a technique described in the appendix by R. D. Barree. The single equation is given by 


$$
R_{\text {eff }}=\frac{8177\left(H_{1} / H_{2}\right)^{1.1648}}{\left(1+\left(\frac{H_{1} / H_{2}}{0.9}\right)^{-9}\right)^{5}}
$$

Equation 19 implies that correlations in the entire range can be represented by power laws connected by transition regions. The logarithmic regime above lies in the 'transition type' region.

The above correlation suggests the following model for proppant transport. Consider some experiment with a channel of given height $H_{2}$ and width $W$. Begin the fluid flow in the channel. After a critical value $Q_{f}$, obtained from equation 14, the fluid begins to move or erode the proppant in the bed. This results in a proppant flow rate corresponding to the given values of $Q_{f}$ and $H_{2}$. The height $H_{2}$ remains almost the same but the depth $H_{1}-H_{2}$ increases as the fluid flow rate is increased. The depth $H_{1}$ to which the bed moves can be found from equation 15. This is Regime 1 or the bed erosion regime. This regime may also be regarded as the bed-load transport regime. The correlation suggests a logarithmic behavior in the bed erosion regime.

Further increase in the fluid flow rate induces suspension of the particles, hence the bed begins to 'inflate' or 'expand'. This situation is identical to our numerical simulations. Bed expansion now causes the value of $H_{2}$ to decrease. This is Regime 2 or the bed expansion regime. As expected from our numerical simulation results, the experimental data also shows power law relation between the fluid Reynolds number and $H_{1} / H_{2}$. The forms of numerical and experimental correlations are not identical obviously due to additional complexities such as three dimensionality and narrow slot in the case of experiments. Nevertheless, we observe that a power law behavior lies at the foundation of these flows.

In order to obtain a fully predictive model another correlation for the proppant flow rate is required. We found that the data correlates best with a non-dimensional variable $R_{p}$ for proppant flow rate given by $R_{p}=\frac{\rho_{f} Q_{p}}{H_{1} \eta}$. The data is plotted in figure 11 . 


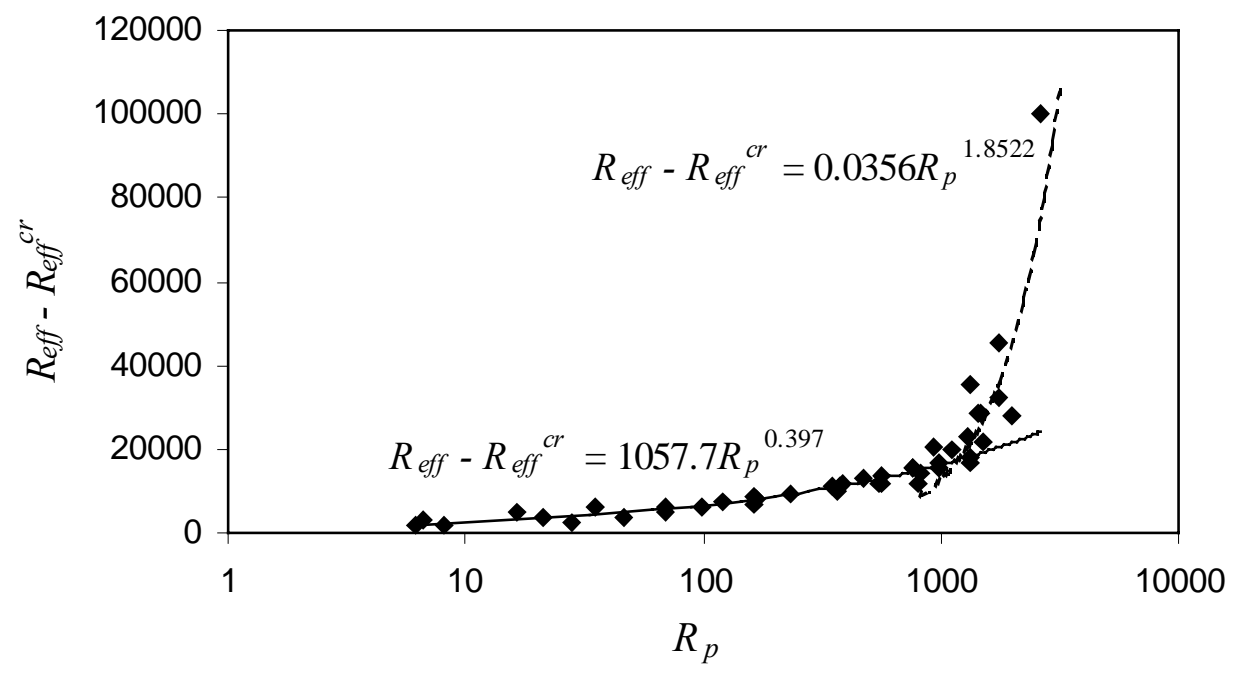

Figure 11. Plot of $R_{e f f}-R_{\text {eff }}^{\text {cr }}$ vs. $R_{p}$.

Once again we observe two regimes consistent with those in figure 10. The correlations are

Bed erosion regime: $\quad R_{\text {eff }}-R_{\text {eff }}^{c r}=1057.7 R_{p}^{0.397}$

Bed expansion regime: $\quad R_{\text {eff }}-R_{\text {eff }}^{c r}=0.0356 R_{p}{ }^{1.8522}$

The transition point is around $R_{\text {eff }}=20000$. Using a technique presented in the appendix by R. D. Barree, equations $20 \& 21$ can be given by a single equation representing power laws connected by transition regions

$$
R_{e f f}-R_{e f f}^{c r}=\frac{1057.7 R_{p}^{0.397}}{\left(1+\left(\frac{R_{p}}{1185.07}\right)^{5}\right)^{-0.291}}
$$

Equations 15-22 represent correlations for proppant transport in a narrow channel. Power laws are observed in agreement with the expectation from numerical results and the analogy between fluidization by drag and lift. The correlations in equations 15-22 can be used as a predictive tool e.g. to obtain the values of $H_{1}$ and $H_{2}$ for given values of fluid and proppant flow rates. A correlation in terms of $\varepsilon$, giving an expression identical to the form in equation 6 , may 
be desirable to develop models for sediment transport for use in simulators. We are currently investigating this aspect further.

Prediction of the transition point between the bed erosion and bed expansion regimes may require further experimental and numerical investigation.

\section{Conclusions}

We believe that research leading to optimal techniques of processing data for correlations from real and numerical experiments is founded on the far from obvious property of self similarity (power laws) in the flow of dispersions. The basis for this belief are the excellent correlations of experiments on fluidization and sedimentation done by Richardson \& Zaki and the correlations for sediment transport in horizontal channels obtained from our numerical simulations and the analysis of the experimental data from STIM-LAB.

Results of two dimensional simulations of solid-liquid flows give rise to straight lines in log$\log$ plots of the relevant dimensionless Reynolds numbers. Power laws are also obtained from the analysis of experimental data. The extent and apparent universality of this property is remarkable and shows that the flow of these dispersions are governed by a hidden property of self similarity leading to power laws. These power laws make a powerful connection between sophisticated high performance computation, experiments and the world of engineering correlations.

The correlations obtained can be used as predictive tools or as a basis for models for sediment transport in simulators used for design purposes.

\section{Acknowledgments}

We acknowledge support by the National Science Foundation KDI/New Computational Challenge grant (NSF/CTS 98-73236), by the DOE, Department of Basic Energy Sciences, by a grant from the Schlumberger foundation, from STIM-LAB Inc. and by the Minnesota Supercomputer Institute. NAP acknowledges the support from Northwestern University through startup funds. 


\title{
Appendix
}

Fitting power-law data with transition regions by a continuous function: Application to the Richardson-Zaki correlation

\author{
R. D. Barree \\ Barree \& Associates LLC
}

Many data sets representing naturally occurring phenomena can be described using a Sigmoidal distribution function. One such function that is particularly useful in fitting physical data is the logistic dose response curve given by:

$$
y=a+\frac{(b-a)}{\left(1+\left(\frac{x}{t}\right)^{c}\right)^{d}}
$$

In this equation each of the constant terms or coefficients $(a, b, c, d$ and $t)$ have readily apparent physical significance, which allows data modeling to be accomplished almost by inspection.

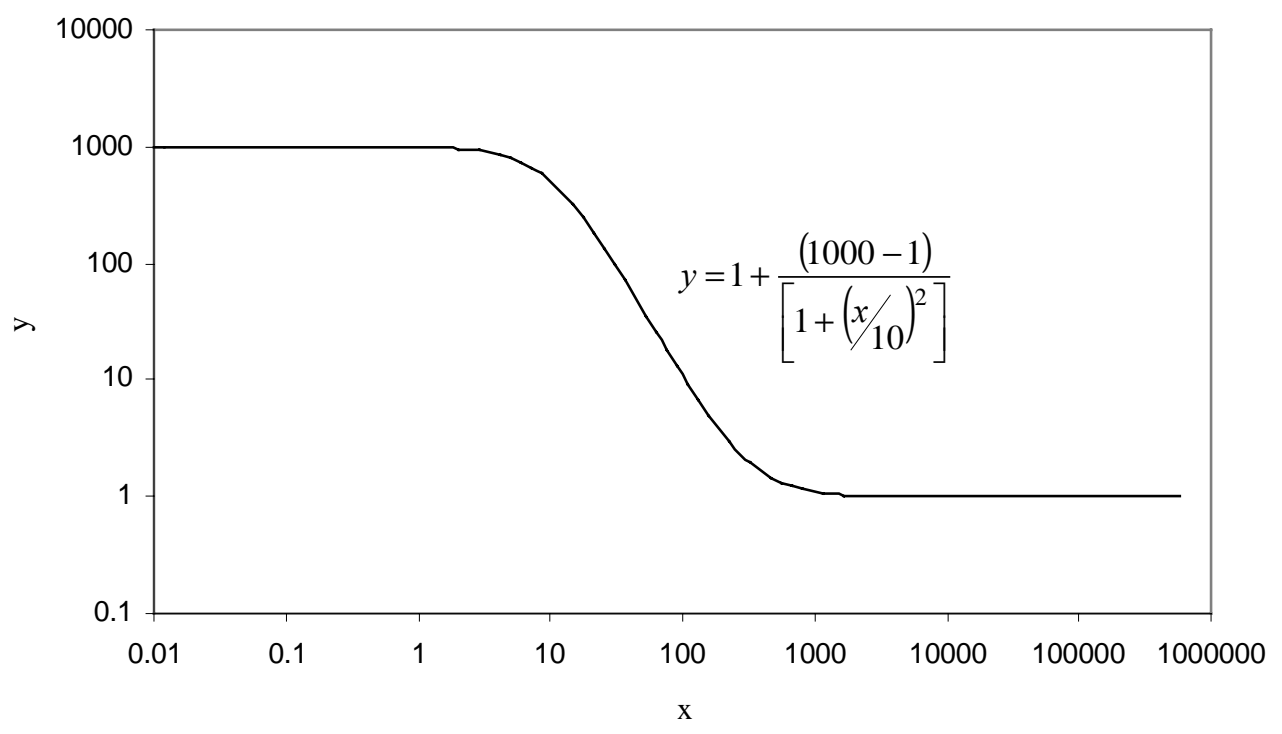

Figure A1. A typical logistic dose response curve.

Figure A1 shows the dose-response function for $a=1, b=1000, c=2, d=1$, and $t=10$. As can be seen, the coefficients $a$ and $b$ represent the values of the lower and upper plateaus of the function, respectively, or its range. The coefficient $t$ defines the value of the independent variable 
$(x)$ where the function deviates from the constant first plateau value. The sharpness of curvature during the deviation from the first plateau is determined by the coefficient $c$. The slope of the power-law straight line in transition from the first plateau to the second plateau is determined by the product of coefficients $c$ and $d$. The slope in this example is negative because both exponents are positive in the denominator of the rational fraction.

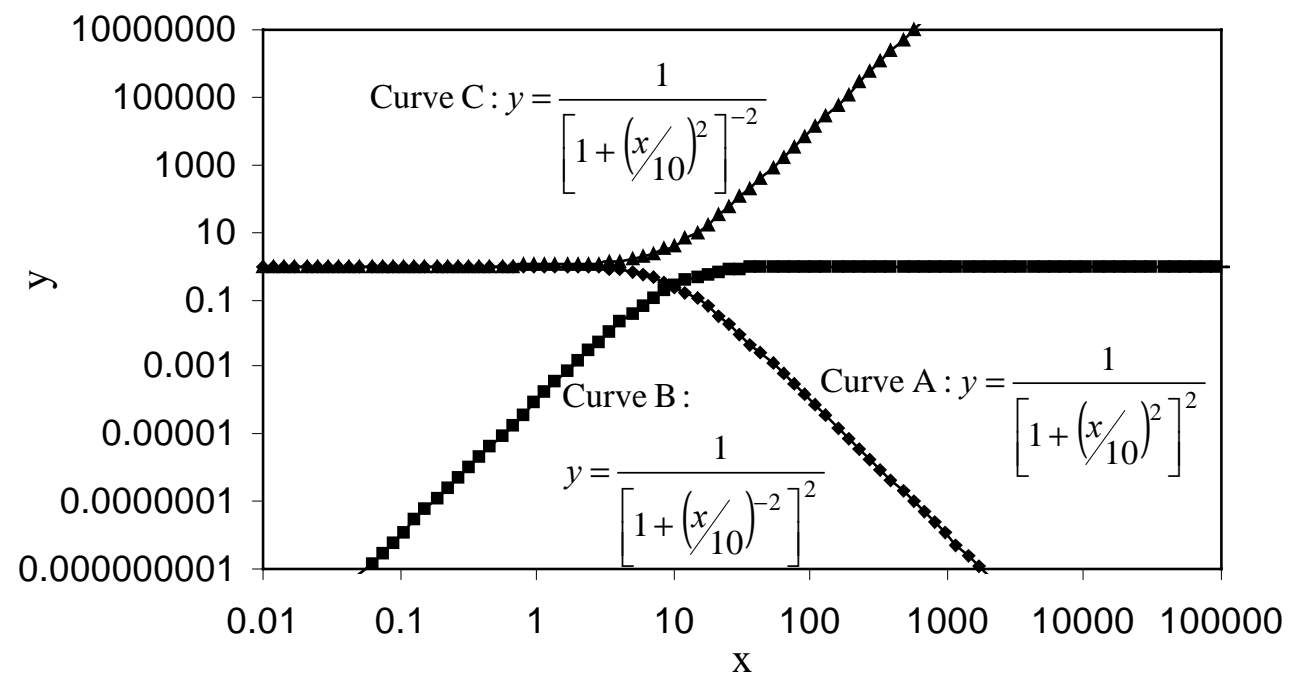

Figure A2. Effects of changing signs of coefficients $c$ and $d$ in the logistic dose response curve.

The effects of changing the signs of the exponents can be examined (figure A2). If the sign of coefficient $c$ is changed, the plot is essentially rotated about a line parallel to the $y$-axis through the transition value $t$ (compare curves $\mathrm{A}$ and $\mathrm{B}$ in figure $\mathrm{A} 2$ ). If the sign of the coefficient $d$ is changed, the plot is rotated about a line parallel to the $\mathrm{x}$-axis through the upper bound $b$ (compare curves $\mathrm{A}$ and $\mathrm{C}$ in figure $\mathrm{A} 2$ ). These relationships allow construction of a transition function in any general form. Another useful property of this function is that the bound corresponding to the coefficient $a$ can be eliminated by setting its value to zero. With $a=0$ the function yields a horizontal line at the upper bound value $b$ and a power-law line of slope $c \times d$ which extends to infinity (Curve A, figure A2). Various functions can then be modeled by products of functions with specified power-law slopes and transition points (figure A3). For curve $\mathrm{A}$ in figure $\mathrm{A} 3 c_{1}=2, d_{1}=1$ and for curve $\mathrm{B} c_{2}=1.6, d_{2}=-1$. The final power-law slope in the product is then $c_{1} \times d_{1}+c_{2} \times d_{2}$. 


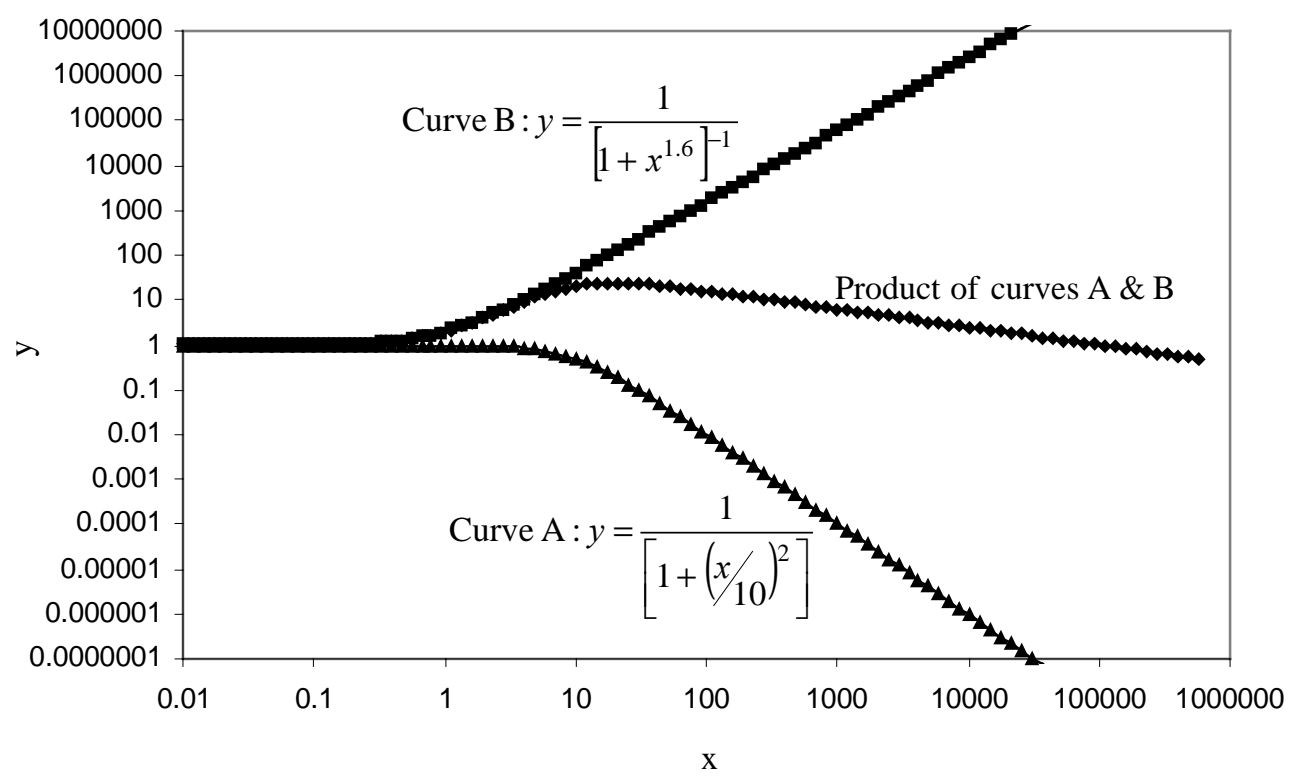

Figure A3. Obtaining a curve from the product of two different logistic dose response curves.

Combinations of these functions can be used in various forms to model many commonly observed phenomena. The logistic dose response curve can also be multiplied by a linear power law function to impose an overall slope to the function. Quite complex systems can be modeled by combining rational fractions or products of multiple functions.

This method has been used to model the Richardson-Zaki correlation that relates bed fluidization velocity to the solids volume-fraction of particles in suspension. The RichardsonZaki correlation is given by equation 1. Specifically, the various functions representing the exponent $n$ are (Richardson \& Zaki, 1954)

$$
\begin{aligned}
& n=\left(4.65+19.5 \frac{d}{D}\right) \text { when } R_{0}<0.2 ; \\
& n=\left(4.35+17.5 \frac{d}{D}\right) R_{0}^{-0.03} \text { when } 0.2<R_{0}<1 \\
& n=\left(4.45+18 \frac{d}{D}\right) R_{0}^{-0.1} \text { when } \quad 1<R_{0}<200 \\
& n=4.45 R_{0}^{-0.1} \text { when } \quad 200<R_{0}<500 ; \\
& n=2.39 \text { when } 500<R_{0}
\end{aligned}
$$


In these relations $d$ is particle diameter, and $D$ is the diameter of the fluidization column. These functions can be replaced with a single continuous form of the logistic dose response curve where $R_{0}$ is the independent variable and $n$ is the dependent result (figure A4).

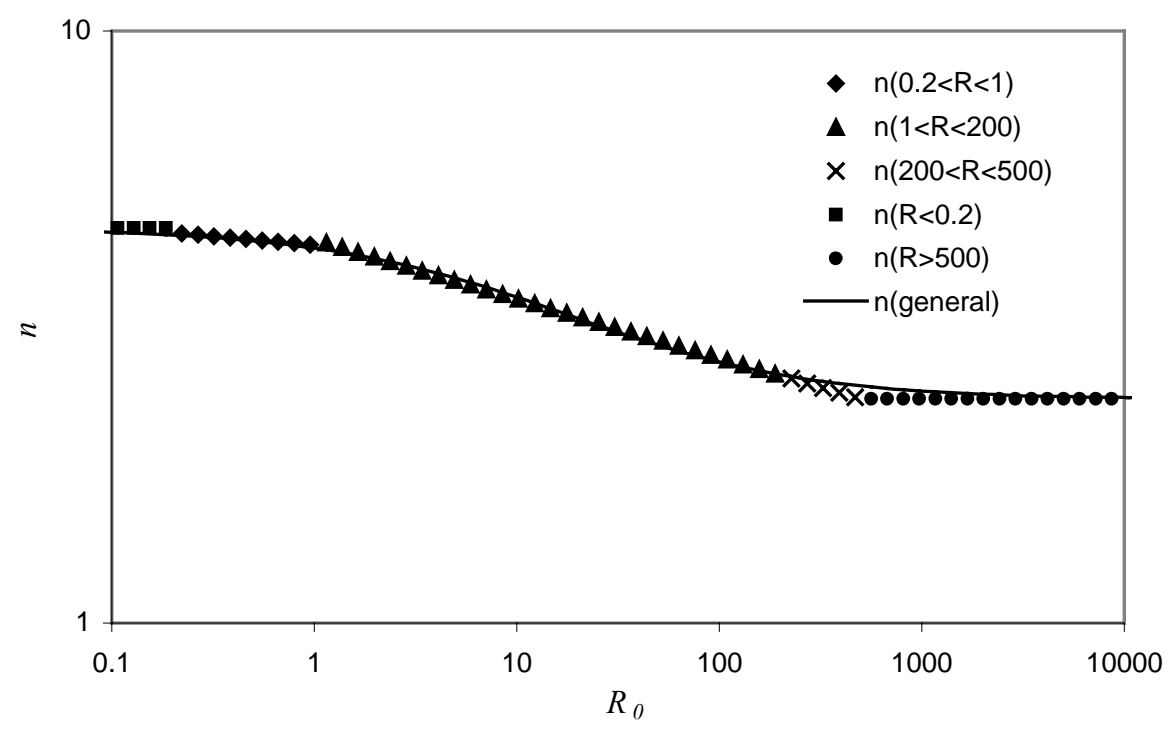

Figure A4. A continuous logistic dose response curve for the Richardson-Zaki exponent $n$ for $d / D=0$.

Figure A4 shows a continuous curve for the Richardson-Zaki exponent $n$. The continuous form of the function is formed assuming that $n$ should not decrease below a value of 2.39 for any value of Re. The continuous form is generated by the equation

$$
n=2.39+\frac{(2.26+19.5 d / D)}{\left[1+\left(\frac{R_{e}}{T}\right)^{0.7}\right]^{1.1}}
$$

This function sets a minimum value of $n=2.39$ and a maximum that is a function of the ratio of particle size to vessel diameter (first of equations A2). The transition value $T$ is also a weak function of the diameter ratio $d / D$, and is given by

$$
T=1+\frac{12.0}{\left(1+\left(\frac{d / D}{0.1}\right)\right)}
$$

The final calculation of $n$ is then given by a combination of equations A3 and A4. 
The same technique has been applied to obtain equations 19 and 22 for proppant transport in horizontal channels.

\section{References}

E.S. Asmolov, 1990 Dynamics of a spherical particle in a laminar boundary layer. Fluid Dyn. 25, 886-890.

E.S. Asmolov, 1999 The inertial lift on a spherical particle in a plane Poiseuille flow at large channel Reynolds number. J. Fluid Mech. 381, 63-87.

T.R. Auton, 1987 The lift force on a spherical body in a rotational flow. J. Fluid Mech. 183, 199218.

R.A. Bagnold, 1966 An approach to the sediment transport problem for general physics. Geological Survey Prof. Paper 422-I, Washington, D.C.

R.A. Bagnold, 1974 Fluid forces on a body in shear-flow; experimental use of 'stationary flow'. Proc. R. Soc. Lond. A. 340, 147-171.

G.I. Barenblatt, 1996. Scaling, Self Similarity and Intermediate Asymptotics. Cambridge Univ. Press.

F.P. Bretherton, 1962 Slow viscous motion round a cylinder in a simple shear. J. Fluid Mech. 12, 591-613.

I. Celik and W. Rodi, 1991 Suspended sediment-transport capacity for open channel flow. $J$. Hydr. Engrg. - ASCE, 117, 191-204.

P. Cherukat, J.B. McLaughlin, and D.S. Dandy, 1999 A computational study of the inertial lift on a sphere in a linear shear flow field. Int. J. Multiphase Flow 25, 15-33.

H.G. Choi, 2000 Splitting method for the combined formulation of fluid-particle problem, to appear in Comput. Meth. Appl. Mech. Engrg.

H.G. Choi and D.D. Joseph, 2001. Fluidization by lift of 300 circular particles in plane Poiseuille flow by direct numerical simulation, accepted, J. Fluid Mech.

A.J. Chorin, 1968 Numerical solution of the Navier-Stokes equations. Math. Comput. 22, 745762.

D.S. Dandy, and H.A. Dwyer, 1990 A sphere in shear flow at finite Reynolds number: effect of shear on particle lift, drag and heat transfer. J. Fluid Mech. 216, 381-410.

D.A. Drew, and S.L. Passman, 1999 Theory of multicomponent fluids, Springer-Verlag, New York. 
R. Eichhorn, and S. Small 1964 Experiments on the lift and drag of spheres suspended in a Poiseuille flow. J. Fluid Mech. 20, 513-527.

P.V. Foscolo, and L.G. Gibilaro, 1984 A fully predictive criterion for transition between particulate and aggregate fluidization. Chem. Engng. Sci. 39, 1667-1675.

A.J. Hogg, 1994 The inertial migration of non-neutrally buoyant spherical particles in twodimensional flows. J. Fluid Mech. 272, 285-318.

H.H. Hu, 1996 Direct simulation of flows of solid-liquid mixtures. Int. J. Multiphase Flow 22, 335-352.

H.H. Hu, and N.A. Patankar, 2001 Simulation of particulate flows in Newtonian and viscoelastic fluids, to appear, Int. J. Multiphase Flow.

H.H. Hu, N.A. Patankar, and M.Y. Zhu, 2001 Direct numerical simulations of fluid-solid systems using the Arbitrary-Lagrangian-Eulerian technique, to appear, J. Comp. Phys.

D.D. Joseph, D. D. 1990 Generalization of the Foscolo-Gibilaro analysis of dynamic waves. Chem. Engng. Sci. 45, 411-414.

D.D. Joseph, 2001 Interrogations of direct numerical simulation of solid-liquid flow. http://www.aem.umn.edu/Solid-Liquid_Flows/, submitted for publication.

D.D. Joseph, D. Ocando and P.Y. Huang, 2001. Slip velocity and lift, accepted, J. Fluid Mech.

T.K. Kern, T.K. Perkins and R.E. Wyant, 1959. The mechanics of sand movement in fracturing, Petroleum Transactions, AIME 216, 403-405.

T. Ko, N.A. Patankar and D.D. Joseph, 2001. A note on the lift-off of a single particle in viscoelastic fluids, submitted, Phys. Fluids.

G.P. Krishnan, and D.T. Leighton, 1995 Inertial lift on a moving sphere in contact with a plane wall in a shear flow. Phys. Fluids 7, 2538-2545.

R. Kurose, and S. Komori, 1999 Drag and lift forces on a rotating sphere in a linear shear flow. J. Fluid Mech. 384, 183-206.

J.B. McLaughlin, 1991 Inertial migration of a small sphere in linear shear flows. J. Fluid Mech. 224, 261-274.

R. Mei, 1992 An approximate expression for the shear lift force on a spherical particle at finite Reynolds number. Int. J. Multiphase Flow 18, 145-147.

J.F. Morris, and J.F. Brady, 1998 Pressure-driven flow of a suspension: buoyancy effects. Int. J. Multiphase Flow. 24, 105-130. 
T.-W. Pan, D.D. Joseph, R. Bai, R. Glowinski and V. Sarin, 2001. Fluidization of 1204 spheres: simulation and experiment, accepted, J. of Fluid Mech.

N.A. Patankar, P.Y. Huang, T. Ko and D.D. Joseph, 2001a. Lift-off of a single particle in Newtonian and viscoelastic fluids by direct numerical simulation, accepted, J. Fluid Mech.

N.A. Patankar, T. Ko, H.G. Choi and D.D. Joseph, 2001b. A correlation for the lift-off of many particles in plan Poiseuille of Newtonian fluids, accepted, J. Fluid Mech.

J.F. Richardson and W.N. Zaki, 1954. Sedimentation and Fluidization: Part I, Trans. Instn. Chem. Engrs. 32, 35-53.

S.I. Rubinow, and J.B. Keller, 1961 The transverse force on a spinning sphere moving in a viscous fluid. J. Fluid Mech. 11, 447-459.

P.G. Saffman, 1965 The lift on a small sphere in a slow shear flow. J. Fluid Mech. 22, 385-400; and Corrigendum 1968 J. Fluid Mech. 31, 624.

A. Shields, 1936 Anwendung der aenlichkeitsmechanik und der turbulenzforschung auf die geschiebebewegung. Mitteilungen der Preussischen Versuchsanstalt fur Wasserbau und Schiffbau, Berlin, Germany, translated to English by W.P. Ott and J.C. Uchelen, California Institute of Technology, California.

J.A. Schonberg and E.J. Hinch 1989 Inertial migration of a sphere in Poiseuille flow. J. Fluid Mech. 203, 517-524.

B.M. Sumer, 1986 Recent developments on the mechanics of sediment suspension. Transport of suspended solids in open channels, Euromech 192, Neubiberg, W. Bechteler, ed., Balkema, Rotterdam, The Netherlands, 3-13.

G. Segré, and A. Silberberg, 1962 Behavior of macroscopic rigid spheres in Poiseuille flow, Part 2. Experimental results and interpretation. J. Fluid Mech. 14, 136-157.

L.C. van Rijn, 1984a Sediment transport, Part I: Bed load transport. J. Hydr. Engrg. - ASCE 110, 1431-1456.

L.C. van Rijn, 1984b Sediment transport, Part II: Suspended load transport. J. Hydr. Engrg. ASCE 110, 1613-1641.

J. Ye, and M.C. Roco, 1992 Particle rotation in a Couette flow. Phys. Fluids A 4, 220-224. 